\title{
Epilog: Zwischen ethnischer und nationaler Identität
}

Das Selbstbewußtsein der deutschen Juden war seit der Aufklärung eng und untrennbar mit der deutschen Staatsbürgerschaft verknüpft. Die formale bürgerliche Gleichstellung war Ausdruck für die Eingliederung der Juden in den deutschen Staat und die Garantie für den Erfolg dieser Integration. Dies setzte bürgerliche Loyalität dem Staat gegenüber voraus. Völlig anders war dagegen die Situation der Juden in Osteuropa. Franz Oppenheimer brachte diese Verhältnisse in seinem 1910 publizierten Aufsatz „Stammesbewußtsein und Volksbewußtsein“ begrifflich auf den Punkt ${ }^{1}$. In dem Versuch, die Differenzen zwischen ost- und westeuropäischem Zionismus zu erklären, unterschied Oppenheimer zwei Begriffe: das Stammesbewußtsein, das sich auf gemeinsame Herkunft, Verwandtschaftsbeziehungen, Protonationalismus sowie eine gemeinsame Leidens- bzw. Siegesgeschichte und kollektive Helden- und Ruhmestaten berufe. Dieses in Deutschland dominierende Bewußtsein unterschied sich Oppenheimers Meinung nach vollkommen von dem Volksbewußtsein, das im osteuropäischen Judentum ausschließlich im Gegenwartsgefühl verankert sei. Oppenheimers Ansicht zufolge hing das Nationalbewußtsein deutscher Juden seiner Zeit an Sprache, Verhaltensmustern, wirtschaftlichen und gesellschaftlichen Beziehungen sowie der geistigen Kultur, also an Elementen, die sie mit der deutschen Gesellschaft teilten. Mit anderen Worten, es war ein durch und durch deutsches Nationalbewußtsein. Ihre Geburtsländer, so erklärte Oppenheimer im Hinblick auf die Juden Westeuropas, hätten ihnen oder ihren Vätern Schutz gewährt und ihnen Sprache, Kleidung, Wohlfahrt und Erziehung, politische Gleichberechtigung und bisweilen auch eine teilweise oder absolute gesetzliche Gleichberechtigung gegeben. Nur ein moralisch verworfener Mensch könne dies vergessen. Jedes Lebewesen schlage dort Wurzeln, wo es lebe. Wer als Bürger in einem etablierten Staat leben könne, sei zu verachten, wenn er darauf nicht mit Loyalität und Dankbarkeit reagiere. Oppenheimer erkannte demnach in den deutschen Zionisten in jeder Hinsicht deutsche Patrioten. Und selbstverständlich trafen seine Definitionen auf die große Mehrheit von Nichtzionisten in Deutschland zu. Die osteuropäischen Juden aber sah Oppenheimer in einem völlig anderen Licht. Sie seien, so meinte er, Juden ihrer Kultur, aber auch ihrer Nationalität nach. Die Rückständigkeit der Staaten, in denen osteuropäische Juden lebten, sowie der Druck, den diese Staaten auf sie ausübten, habe den Juden keinen anderen anständigen Ausweg gelassen, als auf ihrer jüdischen Identität in jedem Sinne zu bestehen. Patriotismus im Hinblick auf den gastgebenden Staat, wie er unter den deutschen Juden zu finden sei, sei in Osteuropa überhaupt nicht möglich.

Die von Oppenheimer für die interne zionistische Diskussion zu Beginn des Jahrhunderts entwickelte Begrifflichkeit kann nun dazu beitragen, die gegenseitigen Beziehungen zwischen deutschem und polnischem Judentum historisch zu verste-

1 Die Welt, F. Oppenheimer, Stammesbewußtsein und Volksbewußtsein, Nr. 7, 18. 2. 1910. 
hen. Die deutschen Juden hatten seit Anfang der sechziger Jahre des 19. Jahrhunderts umfassende Rechte in den deutschen Ländern erlangt, ihre gesetzliche Gleichberechtigung hatte dann zur Zeit der Reichsgründung 1871 eine weitgehende Bestätigung erhalten. Oppenheimer wußte, wie es zu seiner Zeit üblich war, um ein jüdisches "Stammesbewußtsein" - eine ethnische Identität - auch in Deutschland. Er sah jedoch keine Diskrepanz zwischen dem deutschen Nationalbewußtsein der Juden einerseits und ihrem jüdisch-ethnischen Bewußtsein andererseits. Möglicherweise wären diese Identitäten in einer hypothetisch-idealen Situation, also ohne Antisemitismus, überhaupt nicht in ein Spannungsverhältnis oder einen Widerspruch geraten. Der wachsende Antisemitismus jedoch und die kontinuierliche Immigration osteuropäischer Juden setzten die komplexe jüdische Identität einem Interessengegensatz zwischen nationaler und ethnischer Zugehörigkeit aus.

Die entscheidende Weggabelung bei der Definition unterschiedlicher jüdischer Identitäten - deutsch-national einerseits und polnisch-jüdisch andererseits - war der Erste Weltkrieg. Das internationale Denken, das zur Entstehung nationaler Minderheiten mit international anerkanntem Status in den Pariser Vorortverträgen geführt hatte, richtete sich nicht auf die westeuropäischen Staaten, sondern galt allein den neuen Staaten Osteuropas ${ }^{2}$. Deutschland als Nationalstaat war ein westeuropäischer Staat. Deutsche Juden wiesen jeden Versuch zurück, ihre Rechte im Rahmen internationaler Verträge zum Schutze von Minderheiten zu verankern, da dies ihrem Selbstverständnis als gleichberechtigte deutsche Bürger widersprochen hätte. Außerdem gab es keinen eindeutig erkennbaren Grund, deutsche Juden als nationale Minderheit zu definieren. Abgesehen davon, daß sie mit Juden anderer Länder eine ethnische Herkunft und private religiöse Traditionen teilten, besaßen deutsche Juden keine Merkmale, die sie als spezifische Gruppe definiert hätten. In Sprache und Verhalten unterschieden sie sich nicht von ihrer Umgebung, und ihr gesetzlicher Status war identisch mit dem der gesamten deutschen Gesellschaft. Der deutsche Antisemitismus war trotz seines unheilvollen Auftretens kein ausreichender Grund, um Minderheitenrechte zu verlangen. Und vor allem zeigten deutsche Juden, Zionisten wie Liberale, keinerlei Interesse an einem derartigen Status. Der Wunsch, sich als Minderheit zu definieren, ist jedoch hierfür conditio sine qua non ${ }^{3}$.

Deutschland wurde also nach Kriegsende nicht in die Minderheitenverträge eingeschlossen. Doch im Unterschied zu anderen westeuropäischen Staaten wie Frankreich prägte sich in Deutschland keine klare Unterscheidung zwischen Staatsangehörigkeit, ethnischer Herkunft und religiös-kultureller Zugehörigkeit heraus. Die deutsche Staatsangehörigkeit basierte auf ethnischer Herkunft, also auf dem jus sanguinis, und nicht auf dem Territorialprinzip, dem jus soli. Die stete Polemik um den Charakter des deutschen Staates kam unter anderem in den Schwierigkeiten zum Ausdruck, die Gesetz und Bürokratie den Immigranten in den Weg legten, um eine Einbürgerung selbst der Angehörigen der zweiten Generation von Einwandererfamilien zu verhindern ${ }^{4}$. Diese Realität ließ deutsche Juden um so stärker an ihrer

2 Zur Ratio, die der Auffassung von nationalen Minderheiten nach dem Ersten Weltkrieg zugrunde lag, und zu den fatalen Folgen dieses politischen Denkens siehe das faszinierende Kapitel in: Arendt, Totalitarianism, S. $267 \mathrm{ff}$. Siehe auch Diner, Jahrhundert, S. $60 \mathrm{ff}$.

3 Fraenkel, Zionist Policy, S. 21.

4 Brubacker, Staats-Bürger. 
deutschen Staatsbürgerschaft festhalten. Hieran ließen sie nicht rütteln. Und in dieser Haltung lag der Schlüssel zum Verständnis der Beziehungen zwischen deutschen Juden und den in ihren Reihen lebenden Juden polnischer Herkunft.

Die unmittelbar nach dem Ersten Weltkrieg entstandenen Verhältnisse setzten sich in den Jahren der Weimarer Republik fort. Aber auch gegen Ende der Weimarer Zeit - angesichts des Erstarkens der deutschen Rechten und insbesondere des Aufstiegs der NSDAP - wies die weit überwiegende Mehrheit der deutschen Zionisten die Forderung nach Minderheitenrechten für die deutschen Juden zurück ${ }^{5}$. Die politische Wende von 1933 schuf eine völlig neue Situation. Noch bevor sich die Schritte der neuen Regierung abzeichneten, wurde allen Beteiligten klar, daß deutsche Juden zum ersten Mal seit der Emanzipation gezwungen sein würden, unter einem Regime zu leben, das es sich zum Ziel gesetzt hatte, die liberalen Werte zu bekämpfen und die Errungenschaften der Aufklärung aufzuheben. Aus polnisch-jüdischer Perspektive erkannte Moshe Sneh rasch die Konsequenzen. In erster Linie hielt er es für entscheidend, daß deutsche Juden ihre eigene Stellung außerhalb des deutschen Bürgertums erkannten:

„Sie werden gezwungen sein, wider Willen ihre staatsbürgerlichen, wirtschaftlichen und politischen Rechte zu verteidigen. Ideologie und Organisation der Assimilation entsprechen den Bedingungen der völligen Gleichberechtigung, nicht den Bedingungen des Kampfes um die Gleichberechtigung. Die Assimilation wird verschwinden, und Juden werden gezwungen sein, gemeinsam und auf sich allein gestellt nicht mehr die hehren Schlagworte der Freiheit für die Allgemeinheit zu schützen, sondern die eigene Freiheit, ihre eigensten Interessen. Nicht mehr als Vordenker kosmopolitischer Ideen, sondern als Juden ..."6

Genau an diesem Punkt teilten sich die Meinungen innerhalb der Führung des deutschen Judentums. Leo Baeck, der künftig an der Spitze der Reichsvertretung der deutschen Juden stehen sollte, wies die Möglichkeit einer Forderung nach Minderheitenrechten von seiten der deutschen Juden zurück und bestand darauf, es reiche aus, wenn sie ihre grundsätzlichen Bürgerrechte wieder erhielten ${ }^{7}$. Robert Weltsch, der Herausgeber des zionistischen Presserorgans in Deutschland, wandte gegen Baeck und andere ein, sie verstünden nicht, daß die Minderheitenrechte mehr als volle Bürgerrechte seien, aber eben diese auch einschlössen ${ }^{8}$. Allerdings zweifelte er daran, ob deutsche Juden nach dem Januar 1933 überhaupt noch die Möglichkeit hätten, diese Rechte einzufordern.

Im Sommer 1933 kristallisierte sich die Position der deutschen Zionisten hinsichtlich des für Juden im nationalsozialistischen Deutschland erreichbaren Status heraus. Weltsch richtete zu jener Zeit ein von ihm zusammen mit Kurt Blumenfeld verfaßtes Memorandum an die Reichskanzlei Hitlers, in dem indirekt auf die Bereitschaft der Zionisten hingewiesen wurde, eine neue Basis für die separate Existenz einer jüdischen Minderheit in Deutschland zu formulieren9. Aus der internen Korrespondenz der deutschen Zionisten ergibt sich, daß sie ein Festhalten an der Emanzipation letztendlich für zwecklos hielten und ihre Bemühungen daher auf die

5 Einige wenige Ausnahmen waren Nahum Goldmann und Moritz Biliski; siehe Margaliot, Political Reaction, S. 226.

6 Sneh, „Luna nad Berinem“, in: Opinja, 5. 3. 1933, abgedruckt in: Sneh, Writings, S. $287 \mathrm{f}$.

7 YIVO, RG 348, Folder 95, Interview mit Dr. Leo Baeck, Berlin, 11. 6. 1933.

8 YIVO, RG 348, Folder 95, Interview mit Dr. Robert Weltsch, Berlin, 11. 6. 1933.

9 Fraenkel, Zionist Policy, S. 82; Margaliot, Political Reaction, S. 209 f. 
Erlangung eines Minderheitenstatus und einer national-kulturellen Autonomie richteten ${ }^{10}$. Zu jener Zeit waren die Zionisten mit ihrer Position innerhalb der jüdischen Öffentlichkeit in Deutschland noch in der Minderheit.

Die Frage des Status einer nationalen Minderheit für deutsche Juden erweckte innerhalb des organisierten Judentums in Osteuropa großes Interesse, und zwar aufgrund der tiefen Anteilnahme an Fragen jüdischer Nationalität und der Erfahrungen der osteuropäischen Juden am Ende des Ersten Weltkrieges. Emil Margulies tschechischer Zionist polnischer Herkunft und jüdischer Vertreter im Europäischen Nationalitäten-Kongreß - verurteilte die deutschen Juden, die sich weigerten, die Realität richtig einzuschätzen:

„Wir haben in dem vergangenen Jahr darüber gesprochen, ob die deutschen Juden eine nationale Minderheit seien oder nicht. Die deutschen Juden selbst wiesen dies damals zurück [...]. Doch ihnen wurde inzwischen nicht nur die Gleichberechtigung mit den deutschen Staatsbürgern genommen, sondern auch die Gleichberechtigung mit nationalen Minderheiten. Es gibt nur noch ein einziges Volk, das mit einem derartigen Status lebt, das sind die Zigeuner."11

Margulies, aktives Mitglied des Comité des Délégations Juives beim Völkerbund, arbeitete in jenen Monaten an der „Bernheim-Petition“ mit, deren Ziel es war, Deutschland daran zu hindern, die antijüdischen Gesetze in Oberschlesien einzuführen, und es zu zwingen, sich dem Minderheitenvertrag zwischen Polen und Deutschland von 1922 entsprechend zu verhalten. Die Petition war erfolgreich, doch die sich aus ihr ergebenden Konsequenzen blieben allein auf den oberschlesischen Raum beschränkt. Gleichzeitig war eine jüdische Delegation darum bemüht, die Frage der Verfolgung von Juden in Deutschland im Rahmen des Europäischen Nationalitäten-Kongresses in Bern im September 1933 auf die Tagesordnung zu set$z^{2}{ }^{12}$. Dieser Versuch scheiterte, da der Vertreter der deutschen Minderheiten auf der Seite Deutschlands stand und den Antrag zur Aufnahme dieses Themas in die Tagesordnung zurückwies ${ }^{13}$. Als Protest gegen die Position der deutschen Delegation zog sich die jüdische Delegation aus dem Kongreß zurück.

Die Weigerung deutscher Juden, für sich den Status einer Minderheit zu fordern, erwies sich aus osteuropäisch-jüdischer Perspektive als Wiederholung eines althergebrachten Musters, dessen Wurzeln bis zur Ablehnung des Minderheitenstatus durch deutsche Juden im Rahmen der Friedensverträge nach dem Ersten Weltkrieg reichten. Angesichts der Machtlosigkeit der Juden galten Minderheitenverträge, wie sie am Ende des Weltkrieges unterzeichnet worden waren, als sinnvolle Alternative. Der Minderheitenvertrag hätte nach dem Ersten Weltkrieg von allen Staaten implementiert werden müssen, meinte Yitzchak Grünbaum in Reaktion auf die Ereignisse in Deutschland ${ }^{14}$. Rabbiner Isaak Rubinstein, Leiter der Misrachi-Bewegung und Mitglied des polnischen Senats, machte den assimilierten Kreisen des deutschen

10 Vgl. den Brief Martin Rosenbluets an Leonard Montefiori: Margaliot, Political Reaction, S. $211 \mathrm{f}$.

11 Protocole II, S. 57.

12 Interessant ist hier Arendts Bemerkung, die jüdische Delegation beim Minderbeitenkongreß sei nicht befugt gewesen, die Frage der deutschen Juden anzusprechen, da es sich bei deutschen Juden nicht um eine nationale Minderheit handelte: Arendt, 'Totalitarianism, S. 274.

13 Zum Verlauf der Einzelheiten siehe: Landau, Disappointing Alliance, S. $126 \mathrm{ff}$.

14 Haynt, „Die Minderheitenverträge sind in Gefahr" (jidd.), 16. 9. 1934. 
Judentums vor den Delegierten des Jüdischen Weltkongresses im Jahre 1934 schwere Vorwürfe wegen ihrer Überheblichkeit von 1917, als er versucht hatte, sie von der Wichtigkeit der Minderheitenrechte zu überzeugen ${ }^{15}$. Rubinstein ignorierte dabei jedoch die besonderen Bedingungen des deutschen Judentums zu jener Zeit und somit den Umstand, daß damals keine absehbare Notwendigkeit bestanden hatte, für deutsche Juden den Minderheitenstatus zu fordern. Darüber hinaus war es zum Zeitpunkt der Konferenz - also Sommer 1934 - zweifelhaft, ob ein Minderheitenvertrag in der Art, wie er am Ende des Ersten Weltkrieges unterzeichnet worden war, für deutsche Juden noch vorteilhaft gewesen wäre; denn Deutschland war im Oktober 1933 aus dem Völkerbund ausgetreten und hatte sich von allen in entsprechender Form eingegangenen Verpflichtungen ohnehin gelöst. Rubinsteins Vorwürfe waren daher letztlich nicht mehr als Sticheleien.

Die Idee des Status einer nationalen Minderheit fand in den Jahren 1933-1934 Eingang in breite Schichten der jüdischen Führung in Deutschland, wobei die Diskrepanz zwischen Zionisten und Nicht-Zionisten in dieser Frage sich allmählich schloß. Um nicht den Unwillen der Behörden zu wecken und den Eindruck zu vermeiden, deutsche Juden würden das "Weltjudentum" mobilisieren, verzichteten die Zionisten und die Leitung des Central Vereins auf alles, was auf eine Verbindung zwischen ihren Anschauungen und den internationalen Initiativen zum Schutze ihres Status hätte hinweisen können: im Hinblick auf den Jüdischen Weltkongreß, die „Bernheim-Petition“ und auch die Initiative im Rahmen des Kongresses nationaler Minderheiten ${ }^{16}$. Derartige taktische Überlegungen führten zu einer widersprüchlichen Situation: Die Leitung des Central Vereins lehnte jede Lösung im Sinne einer nationalen Minderheit ab, hielt jedoch enge Kontakte zu internationalen jüdischen Körperschaften aufrecht, die auf dieses Ziel hinwirkten, während die deutschen Zionisten sich jeglichen Kontaktes mit den Vertretern des Comité des Délégations Juives enthielten und vorsichtig direkte Kontakte mit den deutschen Behörden suchten. Nur die deutschnationalen Kreise um den Reichsbund jüdischer Frontsoldaten und weitere Randgruppen weigerten sich weiterhin beharrlich, die neue Situation anzuerkennen. Im Sommer 1935 legten die Nürnberger Gesetze die gesetzlich-politische Stellung der Juden im nationalsozialistischen Deutschland als separate Gruppe innerhalb des deutschen Staates fest. Damit aber hatten deutsche Juden keineswegs den Status einer nationalen Minderheit erhalten. Es war jedoch dieser Eindruck entstanden, so daß die Erkenntnis, es müsse dringend eine alternative Lösung für das Leben der Juden in Deutschland gefunden werden, zusätzlich erschwert wurde ${ }^{17}$.

Der Status nationaler Minderheiten, wie er in den Friedensverträgen nach dem Ersten Weltkrieg festgelegt worden war, sollte diese vor dem Mehrheitenstaat schützen und die mangelnde Übereinstimmung zwischen den geopolitischen Grenzen der neuen Staaten und der ethnischen Zugehörigkeit der innerhalb dieser Grenzen lebenden Menschen ausgleichen ${ }^{18}$. Dieser, vom Völkerbund zu garantierende

15 Protocole III, S. $111 \mathrm{f}$.

16 Eine umfassende Darstellung und detaillierte Analyse der Position der politischen Führung des deutschen Judentums im Hinblick auf den Status einer nationalen Minderheit bei: Margaliot, Political Reaction, S. 208-243.

17 Diner, Auswanderung ohne Einwanderung, S. 149.

18 Siehe die kritische Haltung bei: Hobsbawm, Nationen, S. $157 \mathrm{f}$. 
Rahmen sollte ein Hindernis für staatliche Diskriminierungspolitik bilden und gleichzeitig optimale Bedingungen für die Entwicklung interner Autonomie ermöglichen, die als adäquater Ausdruck der sprachlich-kulturellen und religiösen Separation der Minderheit verstanden wurde. Das Fundament dieser Regelungen bildeten jedoch widersprüchliche, ja gefährliche Grundvoraussetzungen ${ }^{19}$ : Sie brachten die offensichtlichen Widersprüche des Nationalstaates zum Ausdruck, deren Wurzeln in der tiefgreifenden Diskrepanz zwischen Staat und Nation lagen; sie waren eindeutiger Ausdruck für die Übertragung des westeuropäischen Staatsverständnisses auf die osteuropäische Realität; sie sollten eine Interimsphase und die notwendige Garantie auf dem Weg zu einer vollständigen Assimilation der nationalen Minderheiten im Nationalstaat darstellen, schaufelten jedoch gleichzeitig das Grab für jede eventuelle Integration der nationalen Minderheiten. Die internationale Anerkennung der Minderheiten betonte praktisch ihre Differenz zur Mehrheit und vertiefte die Beziehungen zu den Angehörigen der jeweiligen nationalen Minderheit in anderen Staaten. In der ersten Phase sah es so aus, als ob Juden in Polen Schutz und gewisse Vorteile durch diese Regelung genießen könnten. Doch dieser Eindruck verlor sich im Laufe der Jahre infolge mangelnder Einigkeit innerhalb des Judentums und durch die tiefer werdenden Zerwürfnisse zwischen den verschiedenen Minderheitengruppen, die Erschütterungen der polnischen Demokratie und letztendlich die Weigerung des polnischen Staates, seinen internationalen Verpflichtungen nachzukommen. Deutsche Juden, die seit der Reichsgründung von 1871 volle staatsbürgerliche Rechte genossen hatten, waren auf den Minderheitenstatus nicht angewiesen. Ihre sprachlich-kulturelle Integration in die allgemeine Gesellschaft machte gleichzeitig die Notwendigkeit und den Wunsch nach kultureller Autonomie überflüssig. Aus ihrer Sicht wäre die Anerkennung als nationale Minderheit ein Rückschritt gewesen, keineswegs Merkmal eines Fortschritts. Hitlers Machtübernahme und die Veränderungen der gesetzlichen Stellung der deutschen Juden hätten den Status einer nationalen Minderheit erneut rechtfertigen können. Doch dies wäre angesichts der Intention der Nationalsozialisten, die Juden völlig aus der deutschen Gesellschaft und dem Staat zu verdrängen, wohl ein unerreichbares Ziel geblieben. Unter derartigen Umständen blieb deutschen Juden nichts anderes übrig, als sich mit dem quasi autonomen Status eines separaten und isolierten Sektors innerhalb der deutschen Gesellschaft zu begnügen, der ihnen vom NS-Regime zugewiesen worden war. Und hier nutzten deutsche Juden trotz fehlender Tradition, eventuell aber infolge ihrer im Vergleich zum polnischen Judentum weitgehend kulturellen und sozioökonomischen Einheitlichkeit, die ihnen zur Verfügung stehenden Rahmenbedingungen und Möglichkeiten effektiv aus.

In den gesellschaftlichen und organisatorischen Konsequenzen, insbesondere aber im autonomen internen Leben der jüdischen Bevölkerung in Deutschland und in dem Umstand, daß sie zu einem geschlossenen, separaten Sektor innerhalb der deutschen Gesellschaft wurden, konnte fortan das ihnen und der jüdischen Gesellschaft in Osteuropa Gemeinsame gesehen werden. Die Blüte der jüdischen Presse und der jüdischen Kultur- und Sportvereine, der Ausbau des jüdischen Schulwesens, der Charakter der Wohlfahrtspflege und viele andere Formen jüdischer Gemeindearbeit weisen darauf hin, daß deutsche Juden sich bemühten, den größtmög-

19 Arendt, Totalitarianism, S. $274 \mathrm{ff}$. 
lichen Nutzen aus der ihnen aufgezwungenen Ghettosituation zu ziehen. Außerdem organisierten sie sich nun rasch in dem zentralen Rahmen der Reichsvertretung der deutschen Juden, einer Körperschaft, die, abgesehen von wenigen isolierten Ausnahmen, die Gesamtheit der deutschen Juden repräsentieren und das interne Leben sowie die Beziehungen zu den staatlichen Behörden regeln sollte. Innerhalb des polnischen Judentums dagegen nahm die Aufsplitterung mit dem Anwachsen des Antisemitismus in Polen erheblich $\mathrm{zu}^{20}$.

Auch dies war ein Paradox, das mit dem offiziellen Status der nationalen Minderheit verbunden war. Eines der auffallendsten Merkmale der politischen Organisierung deutscher Juden war das Fehlen jüdischer Parteien. Die Gründe dafür waren vielfältig: Das sozioökonomische Profil des deutschen Judentums war homogener als das der polnischen Juden - trotz der Diskrepanz zwischen dem mittleren und oberen Mittelstand und der Unterschiede zwischen der urbanen und ländlichen Bevölkerung. Diese relativ große Einheitlichkeit war kein fruchtbarer Boden für die Entstehung politischer Parteien. Hinzu kam die traditionelle Organisierung im Geiste des deutschen Bürgertums, die ihren Ausdruck in den vielfältigen Vereinen der deutschen Juden fand. Zur Gründung jüdischer politischer Parteien kam es eigentlich in keinem Staate, in dem Juden volle bürgerliche Gleichberechtigung genossen. Das Fehlen solcher Parteien in Deutschland weist daher nicht auf eine defizitäre jüdische Organisation hin. Im Gegenteil: Das deutsche Judentum zeichnete sich durch eine reiche organisatorische Tradition aus. Auch wenn deutsche Juden keine lange Tradition der Autonomie, im Sinne der Vierländersynode [Waad arba ha-arazot], kannten, hatte das deutsche Recht ein verpflichtendes Band zwischen der Zugehörigkeit zum Judentum und der Zugehörigkeit zur jüdischen Gemeinde geknüpft und damit die Stellung der jüdischen Gemeinden begründet. Die sozioökonomische Homogenität, das Fehlen einer parteipolitischen Aufsplitterung, die rückläufige Bedeutung der politisch-ideologischen Kämpfe nach der Machtübernahme der Nationalsozialisten, die Existenz eines intakten Gemeindesystems und einer ausgeprägten regionalen und überregionalen organisatorischen Tradition - das alles waren Elemente, die den Weg für die rasche Gründung einer deutsch-jüdischen Dachorganisation nach 1933 geebnet hatten. Diese Entwicklung war nach innen und außen von gewaltiger Bedeutung. Nach innen förderte sie die Auseinandersetzung mit den Maßnahmen des NS-Regimes, indem zentral Informationen gesammelt und ein Netzwerk gemeinsamer Reaktionen und Strategien aufgebaut wurde. Eingeschränkt und gemäßigt wurde zudem die interne politische Aufsplitterung, die innerhalb der deutsch-jüdischen Öffentlichkeit in der Zeit der Weimarer Repubik geherrscht hatte; verbessert wurde die Fähigkeit, von den begrenzten finanziellen Mitteln sinnvollen Gebrauch zu machen. Nach außen ermöglichte der rasche Zusammenschluß im Rahmen der Reichsvertretung die konzentrierte, geordnete Interessenvertretung des deutschen Judentums, um auf diesem Wege die maximale - finanzielle, politische und moralische - Unterstützung der internationalen jüdischen Verbände zu erlangen. Organisatorisch stellte sich das deutsche Judentum nach 1933 als eine in jeder Hinsicht gefestigte Interessengruppe dar.

Das polnische Judentum hatte durch den Versailler Vertrag den offiziellen Status einer Minderheit erhalten. Die praktische Bedeutung dieses Status war äußerst be-

${ }^{20}$ Heuberger, Die jüdischen Parteien; Mendelsohn, East Central Europe, S. 81. 
grenzt und beschränkte sich auf das Anrecht auf jüdische Erziehung und die Einhaltung des Sabbats. Doch da Polen im Prinzip ein multinationaler Staat war, erfolgte die politische Organisation der jüdischen Bevölkerung wie die der anderen Minderheitengruppen auf der Basis politischer Parteien. Während der gesamten dreißiger Jahre war das polnische Judentum in Zionisten, Orthodoxe und Sozialisten mit ihren jeweiligen Parteien zersplittert. Es führte daher einen kontinuierlichen Kampf um die Ausprägung einer politischen Gestalt. Diese tiefe politische Fraktionierung des polnischen Judentums verhinderte die Bildung einer zentralen Körperschaft nach dem Vorbild der Reichsvertretung. Zu dem politischen Kampf kam die organisatorische Schwäche hinzu, die sich in fast allen polnisch-jüdischen Organisationen als großes Übel erwies. Die politische Fraktionierung verhinderte die Bildung einer einheitlichen Front, während die defizitäre Organisation internationale Gruppen von Hilfsaktionen für das polnische Judentum abschreckte. Der Joint hatte den Glauben an die Administrationsfähigkeit polnischer Juden bereits in den zwanziger Jahren verloren, während zionistische Stellen sich häufig über in Polen auftretende Unregelmäßigkeiten bei der Verteilung von Einwanderungszertifikaten nach Palästina beklagte. Angesichts der Umstände, unter denen jeder USDollar und jedes Einwanderungszertifikat von höchster Bedeutung waren, erwies sich die mangelnde Organisationsfähigkeit polnischer Juden als verheerend.

Jüdische Organisationen in Deutschland arbeiteten seit 1933 so, als ob es sich bei der in einem abgeschlossenen separaten Sektor lebenden jüdischen Bevölkerung um eine nationale Minderheit handelte. Doch viele Juden nahmen diese Veränderung nicht bewußt wahr und verstanden ihre Bedeutung nicht. Zahlreiche Widersprüche, auf die die jüdischen Organisationen nach 1933 stießen, ergaben sich aus der Diskrepanz zwischen Wahrnehmung und Realität. Das hartnäckige Verlangen des Centralvereins deutscher Staatsbürger jüdischen Glaubens, ausschließlich die Angelegenheiten deutscher Staatsbürger vertreten zu wollen, die Unsicherheit verschiedener Gemeinden im Hinblick auf die Beschäftigung osteuropäischer Juden in religiösen Ämtern, die überhastete Suche nach vorläufigen Auswanderungslösungen für osteuropäische Juden in Deutschland und der verzweifelte Kampf zwischen den Frauen der in Sachsenhausen internierten Juden und den Vertretern der Reichsvereinigung 1939 - das alles waren Beweise für die Machtlosigkeit jener Körperschaften und Organisationen, adäquate Lösungen für alle Juden in Deutschland zu finden. Gleichzeitig wiesen sie darauf hin, daß das deutsch-jüdische Establishment auch in den dreißiger Jahren die osteuropäischen Juden als Fremde betrachtete, die nicht zur deutschen Nation gehörten. Außerdem bestand die Befürchtung, als Bürger eines anderen Staates seien ausländische Juden eine Bedrohung für den rechtlichen Status deutscher Juden. Nach 1933 gab es allerdings für eine derartige Befürchtung keinerlei Grund mehr. Die Stellung osteuropäischer Juden als Bürger fremder Staaten war im Rahmen bilateraler und internationaler Verträge verankert und in vielerlei Hinsicht nun zunächst der der deutschen Juden vorzuziehen. $\mathrm{Da}$ deutsch-jüdische Organisationen weiterhin in den Begriffen einer rechtsstaatlichen Demokratie verfangen waren, die dem Prinzip der formalen Gleichberechtigung verpflichtet ist und ihre Staatsangehörigen bevorzugt behandeln muß, konnten sie nur schwer die Tatsache akzeptieren, daß sie praktisch Opfer der Diskriminierung geworden waren und ihre Stellung in vielen Bereichen ungünstiger war als die Situation ausländischer Juden. Möglicherweise wurde diese irrtümliche Ansicht zunächst 
noch zusätzlich durch die Ausbürgerung vieler in Deutschland lebender jüdischer Immigranten begünstigt, die zwischen 1918 und 1932 die deutsche Staatsbürgerschaft erhalten hatten. Daneben lebte die traditionelle gesellschaftliche und kulturelle Distanzierung, die sich seit der Masseneinwanderung aus Osteuropa verschärft hatte, ebenso fort wie vereinzelte Annäherungen an die osteuropäisch-jüdische Kultur, insbesondere in den Reihen der Zionisten und der Orthodoxie, die ja auch während der Jahre der Weimarer Republik anzutreffen gewesen waren. Es muß bezweifelt werden, ob der politische Wechsel in Deutschland und die zentrale Rolle des Antisemitismus in der neuen Regierungspolitik dazu führten, daß sich die altansässige deutsch-jüdische Gemeindeführung sich osteuropäischen Juden gegenüber wohlwollender verhielt.

Vor diesem Hintergrund hatten polnische Juden in Deutschland in den jüdischen Gemeinden keinen gleichberechtigten Status gewinnen können. Meistens wurden sie zwar korrekt behandelt - dafür gab es eine lange institutionelle jüdische Tradition in Deutschland. Nach deutschem Recht waren die jüdischen Gemeinden verpflichtet, die Immigranten als Gemeindemitglieder aufzunehmen, es zwang den altetablierten Gemeinden somit eine Bürgschaft auf. Doch in den wirklich wichtigen Fragen im Rahmen der Verhandlungen mit den deutschen Behörden konnten sich die Immigranten nicht auf die Unterstützung der Gemeinden und Landesinstitutionen verlassen. Es bestand eine ausdrückliche Verbindung zwischen dem hartnäckigen Verlangen der Reichsvertretung - in Kooperation mit dem Joint -, polnische Juden in den ersten Jahren nach 1933 nach Polen zurückzuschicken und sich nicht für eine Migration Richtung Westen einzusetzen, und dem Zögern der Reichsvereinigung, die polnischen Internierten von 1939 zu betreuen. Die Reichsvertretung arbeitete nach dem Prinzip der „Repatriierung" und glaubte, es sei vernünftig, die polnischen Juden in ihr Herkunftsland zurückzuführen ${ }^{21}$. Sie richtete das Anrecht der polnischen Juden auf eine Rückführung gegen sie und wahrte damit die Chancen deutscher Juden, nach Westen zu emigrieren. Die Reichsvereinigung zögerte sechs Jahre später, sich für die Freilassung der polnischen Internierten einzusetzen, ihnen Zertifikate für eine sofortige Emigration zur Verfügung zu stellen, und zog es angesichts fehlender Alternativen vor, sich mit der Bitte an die SS zu wenden, die Internierten aus dem Konzentrationslager Sachsenhausen zu entlassen und in die besetzten Gebiete des Generalgouvernements zu deportieren. In beiden Fällen richteten sich deutsch-jüdische Organisationen also nach dem leitenden Grundsatz der „Repatriierung“. Dies war vielleicht der auffälligste Ausdruck einer „deutsch-staatsbürgerlichen“" Position in den Reihen der Führung des deutschen Judentums. Jüdische Führung und Organisationen in Deutschland übertrugen die Bedeutung, die sie der deutschen Staatsangehörigkeit zumaßen, auf die polnische Staatsbürgerschaft der in Deutschland lebenden polnischen Juden. Diese Staatsbürgerschaft gab polnischen Juden zwar einen Anspruch auf gewisse Dienst- und Hilfeleistungen der diplomatischen Vertretungen Polens und ermöglichte ihnen eine Rückkehr nach Polen. Doch im Hinblick auf die Mehrheit der polnischen Juden in Deutschland handelte es sich allein um einen rein formalen Status. Unter den Umständen, unter denen der polnische Staat entstanden war, hatte die polnische Staatsbürgerschaft für polnische Juden nicht die Bedeutung einer Zugehörigkeit

21 Zur Repatriierung im jüdischen Kontext siehe: Weiss, Homeland. 
erlangt. Polen wurde durch den formalen Akt der Verleihung der Staatsbürgerschaft nicht zu ihrem Vaterland, und die Rückkehr nach Polen war in diesem Fall die ungünstigte aller Möglichkeiten.

Es war allerdings während der dreißiger Jahre zu beobachten, wie sich unter polnischen Juden in Deutschland allmählich eine Beziehung zum polnischen Staat entwickelte. Dies kam in einer zunehmenden Organisierung in polnisch-jüdischen Vereinen sowie in den vorsichtigen Äußerungen eines polnischen Patriotismus zum Ausdruck. Außerdem gaben die polnisch-jüdischen Vereine in Deutschland unter polnischem Druck ihre Kontakte zu Mitgliedern ohne polnische Staatsangehörigkeit auf. Nach 1936 vertraten sie ausschließlich polnische Staatsbürger. Dies war nun vielleicht nicht gerade ein Beweis für das Erwachen eines polnischen Patriotismus. Angesichts des wachsenden deutschen Antisemitismus und der besonderen Schwierigkeiten polnischer Juden bei der Emigration nach Westen blieb ihnen letztlich nichts anderes übrig, als sich auf den polnischen Staat zu verlassen. Langfristig gesehen vertrauten sie damit zwar auf ein brüchiges Eis, kurzfristig jedoch reichte es aus, um in Verhandlungen mit den deutschen Behörden Unterstützung zu erhalten. Und selbst die Emigration nach Polen war angesichts einer sich in Deutschland abzeichnenden absoluten Ausweglosigkeit vorzuziehen. Trotz alledem blieben die Beziehungen der polnischen Juden in Deutschland zu Polen pragmatisch und zeugten nicht von einer grundsätzlichen Änderung der Auffassungen.

Die Differenzen zwischen der Interpretation der Staatsbürgerschaft als Identität unter den deutschen Juden und der Staatsangehörigkeit als bloßem von internationalem Recht anerkannten Status unter den Juden Polens beeinflußten das gesamte Beziehungsgeflecht zwischen den jüdischen Bevölkerungen beider Staaten. Hin und wieder wird behauptet, die Integration der deutschen Juden in die deutsche Gesellschaft sei ihnen zum Hindernis geworden und habe es ihnen mehrheitlich erschwert, die neue politische Realität zu verstehen, wodurch sich ihre Suche nach radikalen Lösungen, insbesondere ihre Emigration aus Deutschland, verzögert habe. Im Lichte der hier vorgelegten Befunde sieht es jedoch eher so aus, daß das Gegenteil zu postulieren ist: Die politische Tradition des deutschen Judentums, dem keine jüdische Kollektividentität bekannt war, trug entscheidend zu seiner, unter den Verhältnissen der dreißiger Jahre erfolgreichen Organisation bei. Die jüdische Bevölkerung in Deutschland konnte sich unter anderem deshalb erfolgreich organisieren, weil sie nicht in den Ketten einer kollektiven Auffassung gefangen war.

Berücksichtigt werden muß zudem das Gewicht der jüdischen Öffentlichkeit, genauer: der maßgebenden Meinung von jüdischen Organisationen im Westen, die ausreichend Mittel und Einfluß hatte und die aufgrund gesellschaftlich-kultureller Affinität dem deutschen Judentum stärker zugeneigt war als dem polnischen. Die Organisationsfähigkeit der deutschen Juden erleichterte es vielen Juden im Westen, ihnen finanzielle Sondermittel zur Verfügung zu stellen. Man konnte mit Sicherheit annehmen, daß diese Mittel ihre Ziele erreichten. Die Not der deutschen Juden - im Unterschied zum Leid der Juden in Polen - wurde als akut, nicht als chronisch wahrgenommen. Die den polnischen Juden zugewiesene Unterstützung war eine Frage der Routine, während die Hilfe für deutsche Juden etwas Neues war. Noch schwerwiegender war, daß es bisweilen so aussah, als ob das polnische Judentum ohnehin am Rande des Abgrunds stehe und die andauernde Not zu einer Situation geführt habe, in der jede Hilfe letztlich bedeutungslos war. Demgegenüber garan- 
tierte der relative Wohlstand der deutschen Juden eine effektive und sinnvolle Verwendung der von dritter Seite gewährten finanziellen Unterstützung. In Deutschland hatten Hachscharoth und Berufsausbildungsprogramme noch Sinn, da man hoffen durfte, wenigstens den Jugendlichen werde die Emigration gelingen. Im Hinblick auf die dreieinhalb Millionen Juden in Polen konnte man die Auswanderung nur mit Schwierigkeiten als allumfassende Lösung in Betracht ziehen.

Deutsch-jüdisches Kapital, hohe berufliche Qualifikation und besondere finanzielle Zuwendungen zugunsten der deutsch-jüdischen Emigration führten letztlich zu einer Bevorzugung deutscher Juden bei der Einwanderung nach Palästina. Die Chancen deutscher Juden, nach Westen zu emigrieren, waren ohnehin stets größer gewesen als die entsprechenden Möglichkeiten polnischer Juden. Schon in jenen Tagen hatten sich etliche Stimmen gegen die diskriminierende unterschiedliche Behandlung von deutschen und polnischen Juden erhoben und gewarnt, die Bevorzugung der deutschen Juden könnte die notwendigen Initiativen zur Verbesserung der Lage polnischer Juden direkt und negativ beeinflussen. Rückblickend betrachtet war dies von Bedeutung für die Rettung der Juden in Polen und Deutschland, in den dreißiger Jahren jedoch ging es allein um die Schaffung erträglicher Lebensbedingungen.

Unter den seit der Emanzipation entstandenen historischen Umständen hatten Juden in Westeuropa Gleichberechtigung genossen. Gleichzeitig hatten sie sich für den Schutz des Status der Juden in Ländern eingesetzt, in denen diese offen diskriminiert wurden. Dabei hatte es sich in erster Linie um die Länder Osteuropas gehandelt. Der Aufstieg des Nationalsozialismus in Deutschland kehrte die Verhältnisse um. In gewissem Maße bildeten polnische Juden nun eine Lobby zur Bewahrung bzw. Wiederinkraftsetzung der Rechte deutscher Juden. Sie versuchten, die jüdische Öffentlichkeit zu mobilisieren und zum Kampf gegen die vom Nationalsozialismus für alle Juden Europas ausgehenden Gefahren aufzurufen. Aus ihrer Position als gleichberechtigte Bürger in einem multinationalen Staat agierten Juden in Polen auf allen Ebenen: durch jüdische Vertreter in Senat und Sejm, auf den Rednertribünen der Massenversammlungen, in der jüdischen Presse und an der Spitze der Boykottbewegung gegen deutsche Waren. Dies war in vielen Fällen ein heroisches Verhalten und verlangte manches materielle Opfer, etwa für die polnischjüdischen Flüchtlinge aus Deutschland.

Deutsche Juden führten keinen vergleichbaren Kampf. Unter der Drohung des NS-Terrors waren ihre Hände gebunden. Darüber hinaus fehlte es ihnen im Unterschied zu polnischen Juden an einem ausdrücklich kollektiven Bewußtsein, und sie vertrauten nicht auf einen umfassenden Kampf. Moshe Kleinbaum (Sneh) faßte Ende 1938 eine weithin verbreitete Kritik polnischer Juden an den Juden in Deutschland mit den Worten zusammen:

„Das deutsche Judentum ist bis auf den Grund verdorben. Die Art und Weise, in der deutsche Juden die Verfolgungen, die Erniedrigungen, die Beleidigungen aufnehmen, das Maß an Kapitulation, mit dem sie auf die abscheulichen Taten des Nazi-Regimes reagieren, das alles belegt das Fehlen von Eigenstolz, den Mangel an geistiger Kraft und moralischem Rückgrat. Wo sind die kleinen Zeichen für einen Widerstand unter deutschen Juden? Sicherlich, ein derartiger Widerstand kann gar nicht effektiv sein, kann keinen Erfolg erzielen. Aber wie können Menschen diese inhumane Behandlung ertragen, ohne ein Zeichen von Protest zu zeigen, von Trotz, von verzweifeltem Widerstand (selbst wenn er wenig zweckdienlich ist?!) Warum gelang es dem russischen Judentum in der Zarenzeit trotz alledem, hier und dort Widerstand zu zeigen? 
Warum brachte das russische Judentum Revolutionäre und Attentäter hervor, die sich selbst als Märtyrer opferten? Weil sie das Judentum in ihrer Seele trugen, weil sie die Bedeutung des Leidens verstanden hatten, weil sie ihr jüdisches Schicksal in allen Tiefen durchlebt hatten. Die deutschen Juden sind dagegen jedem jüdischen Inhalt entfremdet. Sie nehmen ihre Katastrophe als persönliches Schicksal von Individuen an, weil ihnen das Gefühl der Zugehörigkeit zum jüdischen Kollektiv fehlt, das Gefühl der jüdischen Schicksalsgemeinschaft. "22

Sneh schränkte seine Anschuldigungen zwar sogleich ein, indem er das Ausbleiben jüdischen Widerstands in den Kontext des Fehlens jeglichen Widerstandes in Deutschland und der Schwäche der Juden als kleinster Minderheitengruppe stellte. Doch es wird deutlich, daß die charakteristischen Merkmale jüdischen Verhaltens in Deutschland, wie sie bei Sneh dargestellt werden, sehr wohl mit der deutsch-jüdischen Tradition verknüpft waren. Das Festhalten am Gesetz, auch als dieses Gesetz deutsche Juden nach 1933 auf Schritt und Tritt diskriminierte, war ein tragender Eckstein der deutsch-jüdischen Geschichte. Illegale Aktivität, so wenig effektiv sie vielleicht auch gewesen sein mag, gehörte nicht zum Repertoire politischer Kultur deutscher Juden. Sie nahmen auch das große Interesse, das osteuropäische Juden der Situation deutscher Juden entgegenbrachten, mit wenig Wohlwollen auf. Die jüdische Presse in Deutschland hatte zwar stets die antisemitischen Tendenzen in Osteuropa verfolgt - aufgrund eines ehrlichen Interesses und als legitime oder gefahrlose Möglichkeit, öffentlich eine freie Diskussion über den Antisemitismus zu führen. Doch von hier bis zur Formulierung einer allgemeinen jüdischen Strategie im Kampf gegen den Antisemitismus blieb es ein weiter Weg.

Praktisch zogen deutsche Juden eindeutige Vorteile aus der Linie, die die zentralen jüdischen Organisationen nach 1933 einschlugen. Sie vergeudeten keine Zeit mit ohnehin chancenlosen und vielleicht gefährlichen prinzipiellen Machtdemonstrationen. Sie boykottierten Deutschland nicht, sondern stellten sich gegen den Boykott. Tausende versuchten - erfolgreich - mittels des Transfer-Abkommens ihren Besitz und ihr Vermögen zu retten und nach Palästina zu emigrieren. Sie gewöhnten sich rasch an die Notwendigkeit der Emigration. Ihre Führung unterstützte mittels eines organisierten Emigrationsapparates, einer professionellen Umschulung und sozialer Hilfe die beschleunigte Auswanderung und sorgte für die in Deutschland zurückbleibenden Personen. Deutsch-jüdische Organisationen verzichteten bisweilen auf ihren Stolz und trugen zu einer Beruhigung der Gemüter unter der internationalen jüdischen Öffentlichkeit bei, wenn sie von den deutschen Behörden dazu aufgefordert wurden. Gleichzeitig bemühten sie sich um praktische Lösungen zur Verbesserung ihrer Situation. Sie versuchten unermüdlich, Gelder zur Linderung der Not aufzutreiben, und achteten wenig auf die indirekten Auswirkungen der "Lösung der Judenfrage" in Deutschland auf die "Judenfrage" in den Ländern Osteuropas. Dies entsprach der von vielen deutschen Juden akzeptierten Auffassung, nicht in kollektiv-jüdischen Begriffen zu denken. Im nachhinein erwies sich diese Haltung als richtig - vor allem im Hinblick auf die zu erzielende Effektivität, denn bis zum Ausbruch des Zweiten Weltkrieges konnten sehr wohl Teillösungen für die separate Not deutscher Juden gefunden werden, insbesondere wenn ihre Situation nicht mit dem Leid der Juden in Polen verknüpft wurde.

22 Der Tag, M. Sneh, Widerstand und Aufbau, 23. 12. 1938, abgedruckt in: Sneh, Writings, S. 312. 